
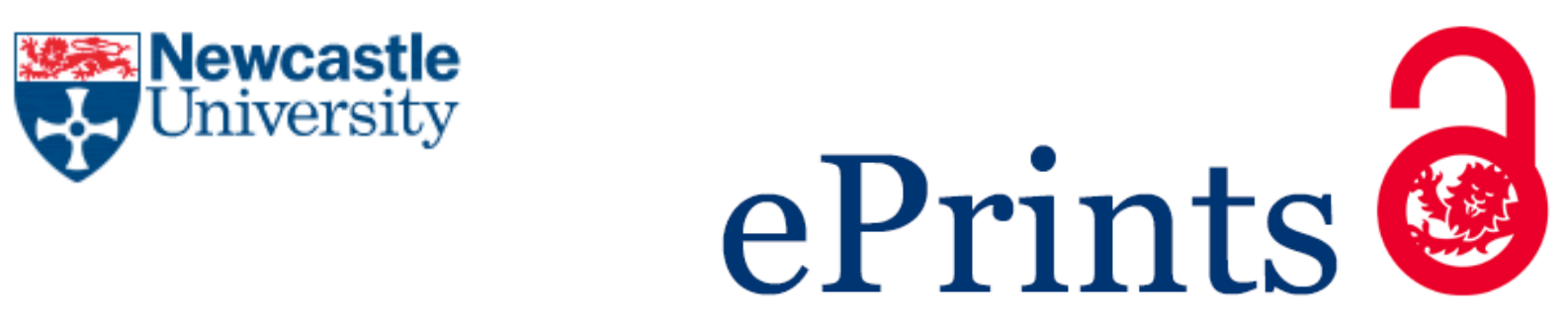

Wells GH, Hunt MRC, Hopf T, Vassilevski K, Escobedo-Cousin E, Horsfall AB, Goss JP, O'Neill A. Facile technique for the removal of metal contamination from graphene. Journal of Vacuum Science \& Technology B 2015, 33(5), 051802.

Copyright:

(C) 2015 American Vacuum Society.

DOI link to article:

http://dx.doi.org/10.1116/1.4928422

Date deposited:

$20 / 08 / 2015$

Embargo release date:

11 August 2016

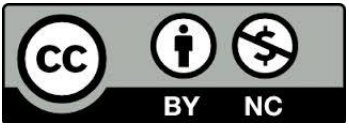

This work is licensed under a Creative Commons Attribution-NonCommercial 3.0 Unported License 


\title{
A Facile Technique for the Removal of Metal Contamination from Graphene
}

\author{
G. H. Wells ${ }^{\mathrm{a})}$ and M. R. C. Hunt ${ }^{\mathrm{b})}$ \\ Centre for Materials Physics, Department of Physics, University of Durham, DH1 3LE, \\ United Kingdom \\ T. Hopf, K. V. Vassilevski, E. Escobedo-Cousin, A. B. Horsfall, J. P. Goss, and A. G. O'Neill \\ School of Electrical and Electronic Engineering, Newcastle University, Newcastle upon Tyne, NE1 7RU, \\ United Kingdom
}

(Dated: 23 July 2015)

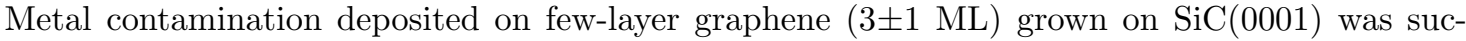
cessfully removed from the surface, using low cost adhesive tape. More than $99 \%$ of deposited silver contamination was removed from the surface via peeling, causing minimal damage to the graphene. A small change in the adhesion of graphene to the $\mathrm{SiC}(0001)$ substrate was indicated by changes observed in pleat defects on the surface, however, atomic resolution images show the graphene lattice remains pristine. Thin layers of contamination deposited via an electron gun during AES/LEED measurements were also found to be removable by this technique. This contamination showed similarities to "roughened" graphene previously reported in the literature.
\end{abstract}

\section{INTRODUCTION}

The excellent electronic properties of graphene and its outstanding sensitivity to adsorbed molecules have resulted in its use in the development of sensor technology $y^{1-3}$. However, these technological applications require graphene layers to be pristine and uncontaminated. With the production of graphene films now common ${ }^{4-6}$, preventing or reducing contamination is now a key issue. It has been shown that the presence of surface contamination has a considerable effect on graphene's electronic properties. Charged impurities in particular cause significant degradation to electron transport due to scattering from the inhomogeneous electron density at these points ${ }^{7-9}$. Larger scale contamination has been shown to change the electronic characteristics of entire graphene films due to doping ${ }^{2}$. Furthermore, graphene's mechanical properties also degrade; the wettability of graphene was found to decrease significantly upon becoming contaminated from long exposure to the atmosphere ${ }^{10}$. In addition to adsorption of impurities from the atmosphere, contamination can occur from other sources. The process used to transfer CVD grown graphene has been shown to introduce a significant amount of metallic contamination which subsequently alters its electrochemical properties to a large degree $\mathrm{e}^{11-13}$. Electron beam filaments associated with device production or characterisation techniques can also deposit material on the graphene surface ${ }^{2}$. These sources are often more problematic due to contamination occurring over large areas, rather than as individually adsorbed molecules. Unlike atmospheric contamination, the impurities deposited during experimental processes are often

\footnotetext{
a) Electronic mail: g.h.wells@durham.ac.uk

b) Electronic mail: m.r.c.hunt@durham.ac.uk
}

metallic and bond more strongly to the surface preventing their removal via annealing ${ }^{14,15}$.

With contamination of graphene samples occurring due to many different factors, which are often unavoidable, cleaning of graphene is important. As a result, many different cleaning methods have been reported including mechanical sweeping with an atomic force microscope (AFM) tip ${ }^{16,17}$, the use of sacrificial metal layers ${ }^{18}$, the application of high currents ${ }^{19}$ and high temperature annealing under vacuum ${ }^{14,15}$. Although these methods are appropriate for the removal of small concentrations of impurities they are less suitable for cleaning thicker contaminant layers over larger scales.

In this work, we report the use of adhesive tape to remove metal contamination. We apply tape to graphene samples grown on $\mathrm{SiC}(0001)$ substrates, which have been coated with a layer of silver which acts as a model metal contaminant. Contamination adheres to the tape and is removed upon peeling away from the substrate, leaving pristine graphene on the surface. We show this procedure is an effective method to remove large areas of noble metal contamination without the use chemicals or specialized equipment allowing for the reuse of graphene for electronics purposes. Furthermore, we have found that material previously characterised as "rough" graphene ${ }^{20}$, may actually be associated with contamination deposited from an electron gun, which can also be removed via this technique.

\section{EXPERIMENT}

Few layer graphene samples $[3 \pm 1$ monolayers $(\mathrm{ML})]$ were grown on $6 \mathrm{H}-\mathrm{SiC}(0001)$ wafers (Tankeblue Semiconductor Co. Ltd.). The growth process was performed in an upgraded commercial rapid thermal processor with a background pressure of $<3 \times 10^{-6}$ mbar at $\mathrm{T}<1900^{\circ} \mathrm{C}$. 


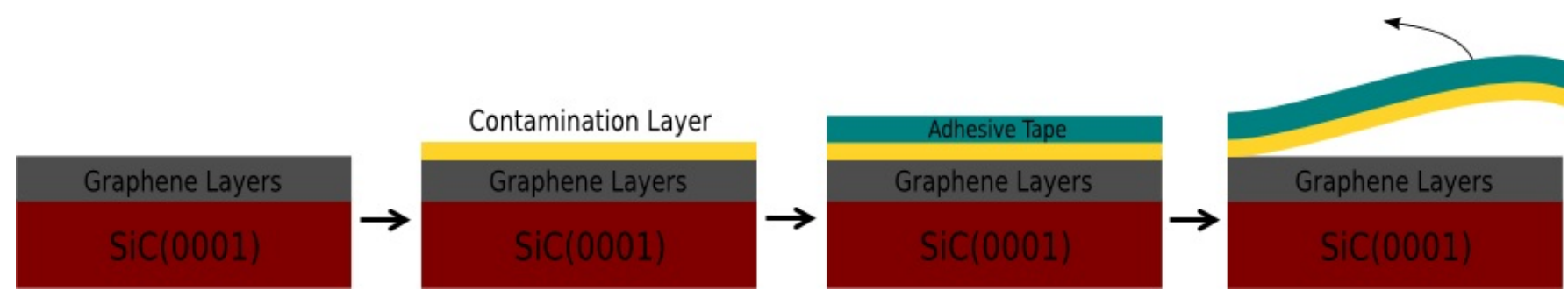

FIG. 1. (Color online) Experimental process of contamination deposition followed by removal by adhesive tape.

Substrates were prepared in-situ by etching in $5 \% \mathrm{H}_{2} / \mathrm{Ar}$ forming gas at atmospheric pressure, followed by a 20 minute heating step at $1200^{\circ} \mathrm{C}$ before ramping to the growth temperature of $1850^{\circ} \mathrm{C}$. Full details of the growth procedure and characterization are reported elsewhere ${ }^{21}$. Samples were transferred, through air, to an ultra-high vacuum (UHV) system (base pressure $<5 \times 10^{-10}$ mbar) and gently annealed for 4 hours at $200^{\circ} \mathrm{C}$, to remove any atmospheric contamination from the surface that might have occurred during transfer to the UHV system. Scanning tunnelling microscopy (STM) was used to image samples before silver deposition. $99.99 \% \mathrm{pu}-$ rity silver (Agar Scientific) was evaporated via heating a wrapped tungsten filament to $\sim 960^{\circ} \mathrm{C}$, to act as a model source of contamination. Evaporation was performed for 10 minutes to produce thick Ag layers, whilst a shorter time of $30 \mathrm{~s}$ was used for lower coverages. Samples were re-imaged via STM to determine the thickness of the Ag layer, then removed from the system and cleaned using commercially available Scotch Magic Tape. The adhesive tape was applied directly to the sample under ambient conditions and peeled away unidirectionally, as shown schematically in figure 1. After cleaning the graphene was reintroduced to the UHV system and immediately examined again, without further cleaning or annealing, by STM, low energy electron diffraction (LEED) and Auger electron spectroscopy (AES). STM measurements were performed using a commercial Omicron VT-SPM system with all images obtained in constant current mode. AES measurements, taken at a beam energy of $\mathrm{E}=2.5 \mathrm{keV}$, were used to obtain an estimate of the graphene thickness based on the $\mathrm{C} K L L$ and $\mathrm{Si}$ $L M M$ peak height ratio as reported previously ${ }^{20,22}$.

\section{RESULTS AND DISCUSSION}

Figure 2(a) shows graphene grown on $\mathrm{SiC}(0001)$ on which well defined terraces and small irregular islands are clearly visible. Pale lines visible on the surface are pleat defects that form during growth, due to the difference in thermal expansion between the graphene layers and the $\mathrm{SiC}$ substrate ${ }^{22,23}$. The high quality of the graphene samples before contamination is further illustrated by the LEED pattern showing clear graphene, $\mathrm{SiC}(1 \times 1)$ spots and $(6 \sqrt{3} \times 6 \sqrt{3}) R 30^{\circ}$ spots corresponding to the surface reconstruction known to form when graphene grows via Si sublimation ${ }^{6}$ [Fig. 2(c)]. After 10 minutes of deposition a Ag layer of $14 \mathrm{~nm}$ thickness, had been deposited on the graphene, covering $95 \%$ of the surface. The deposited layer is not uniform, having an root mean squared (RMS) roughness of $1.4 \mathrm{~nm}$, with some larger areas where the graphene surface can be observed below, as can be seen in Fig. 2(b) where the edge of a terrace and a pleat defect are visible beneath the contamination. The large thickness and non-uniformity of the Ag layer effectively simulates the contamination of a sample due to uncontrolled deposition.

Figure 2(d) shows the graphene sample after the removal of contamination. Clear graphene terraces and small isolated islands are once again visible on the surface. A small amount of what is likely to be trace silver is still visible in the lower left corner. From further images of the surface this appears to be a small isolated area. It is apparent that $\sim 99 \%$ of the $\mathrm{Ag}$ contamination has been removed by the tape. Furthermore, the cleaning process appears to cause very little damage to the surface with only small areas visible in which tiny flakes of graphene have been pulled away from the substrate. One significant difference that is observed between images taken before and after contamination and cleaning is in the properties of pleat defects on the surface. Pleat defects on graphene grown on $\mathrm{SiC}(0001)$ are known to form during growth due to the differential thermal expansion between the graphene layers and the substrate, forming along high symmetry directions of the lattice ${ }^{22}$. After the cleaning process it was found that the concentration of pleat defects on the surface increased from $13 \pm 3 / \mu \mathrm{m}^{2}$ to $19 \pm 3 / \mu \mathrm{m}^{2}$. Pleats were also found to increase in length after cleaning by an average of $81 \mathrm{~nm}$; increases in pleat height combined with a reduction in width were also observed, as can be seen in the cross-sectional profiles in Fig. 3. Despite overall increases in pleat length, height and concentration it was also observed that the variation in these values also significantly increased after the contamination was removed. This suggests that the cleaning process has caused pre-existing pleat defects to further delaminate from the surface, whilst also inducing new pleats to form, indicating that the adhesion of the graphene layers to the underlying $\mathrm{SiC}$ substrate has been altered to a degree, due to the film being strained whilst cleaned. Despite these changes, the graphene remains 

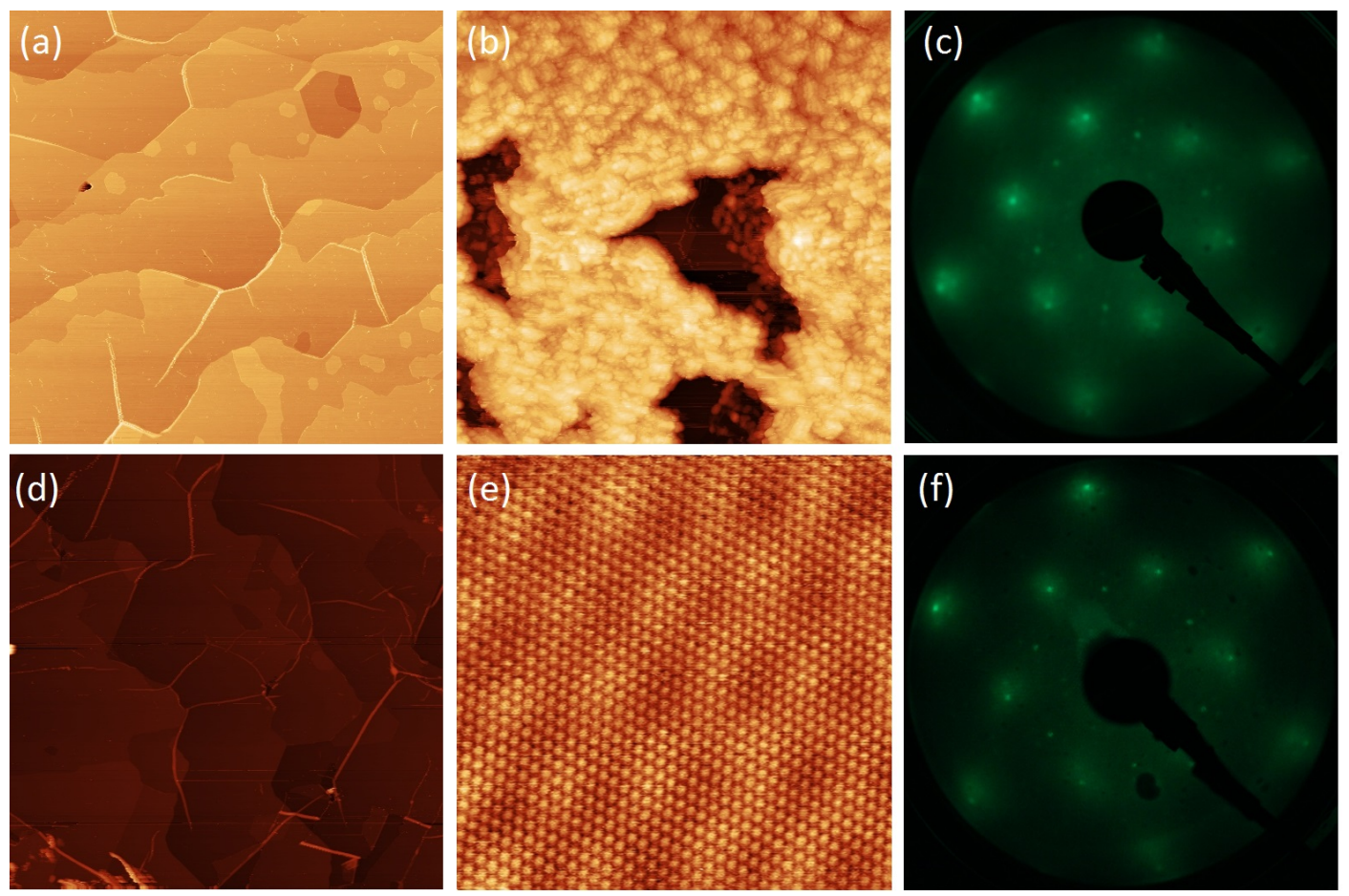

FIG. 2. (Color online) (a) $1 \mu \mathrm{m}^{2} \mathrm{STM}$ image of uncontaminated graphene grown on $\mathrm{SiC}\left(\mathrm{V}_{\text {bias }}=1.1 \mathrm{~V}, I=0.5 \mathrm{nA}\right)$. (b) $1 \mu \mathrm{m}^{2}$ image of the graphene sample contaminated via $\mathrm{Ag}$ evaporation $\left(\mathrm{V}_{\text {bias }}=2.2 \mathrm{~V}, I=0.4 \mathrm{nA}\right)$. (c) LEED pattern of the graphene before contamination, at beam energy $E=180.2 \mathrm{eV}$, showing graphene and $\mathrm{SiC}(1 \times 1)$ and $(6 \sqrt{3} \times 6 \sqrt{3}) R 30^{\circ}$ reconstruction spots. (d) $1 \mu \mathrm{m}^{2}$ of graphene after cleaning via mechanical exfoliation by adhesive tape $\left(\mathrm{V}_{\text {bias }}=2.0 \mathrm{~V}, I=0.3 \mathrm{nA}\right)$. (e) $12 \mathrm{~nm}{ }^{2}$ atomic resolution image of the pristine graphene lattice and buffer layer reconstruction pattern, after contamination and cleaning $\left(\mathrm{V}_{\text {bias }}=-0.2 \mathrm{~V}, I=1.6 \mathrm{nA}\right)$. (f) LEED pattern of the graphene after contamination and subsequent cleaning at beam energy $E=181.1 \mathrm{eV}$, showing identical spots to those observed before contamination.

of a high quality as seen in Fig. 2(d), further confirmed by Fig. 2(e) which shows the pristine hexagonal lattice, which was consistently observed at multiple points across the surface. The LEED pattern obtained after cleaning also attests to the high degree of graphene cleanliness and perfection, showing no visible change from that obtained before $\mathrm{Ag}$ deposition, with clear graphene, $\mathrm{SiC}$ and reconstruction spots [Fig. 2(f)].

To explain the removal of contamination from the surface leaving the graphene in pristine condition, we propose that the Ag contamination adheres to the graphene surface weakly. This is most likely due to its inert nature which has prevented growth of graphene on silver substrates until recently ${ }^{24}$. In contrast, the adhesion of graphene to $\mathrm{SiC}(0001)$ is relatively strong in comparison to graphene on most other substrates ${ }^{22,25,26}$. Therefore, when tape is applied to the contaminated sample the silver adheres more strongly than to the graphene film. As a result, when peeled away from the surface the contamination is also removed, whilst the stronger adhesion of the graphene to the substrate ensures the film remains adhered to the surface. It can be surmised that in areas that are still free of contamination after deposition, the tape may adhere more strongly to the uppermost layer than the graphene adheres to the substrate. As a result

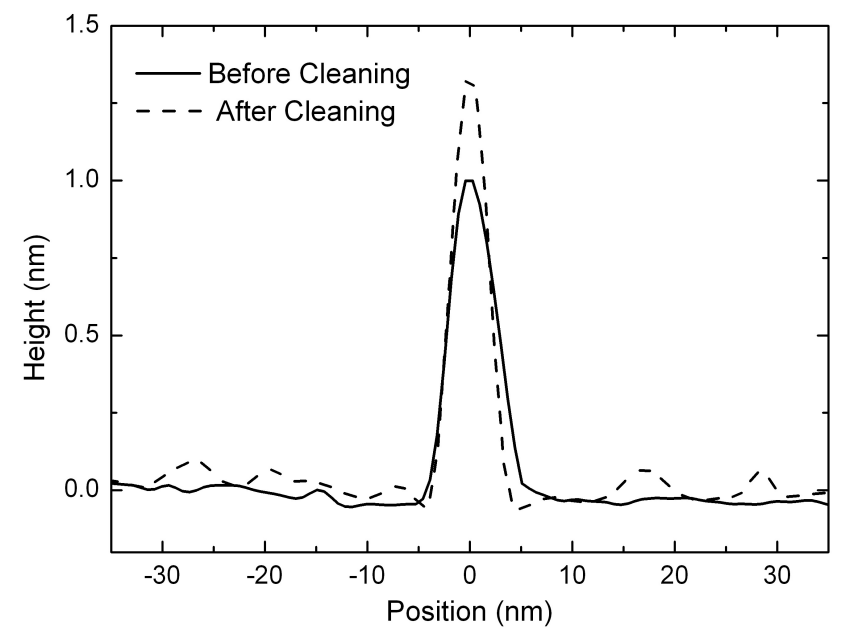

FIG. 3. Cross section height profile of typical pleat defects before and after contamination removal. After cleaning pleats are generally taller and narrower than those measured before contamination.

we would expect to see a high defect concentration in these areas; however, this was not observed. We propose that the tape adheres primarily to the raised asperities 
offered by contamination on the partially covered surface, rather than conforming to the surface topography. Due to the high flexibility and mechanical strength of graphene $^{27}$, adhesion over a large area is likely required to delaminate and/or damage the uppermost graphene layer. Hence, the concentration of defects produced by the cleaning method remains low. The small areas of contamination that remain on the surface after cleaning can be explained by defects in the graphene lattice prior to deposition which create reactive sites to which contamination adheres more strongly ${ }^{28}$.

With the removal of the silver contamination and a pristine surface observed below, a possible alternative explanation to be considered is that mechanical exfoliation pulls off both the Ag contamination and the uppermost graphene layer the silver is adhered to, similar to the first experimental production of isolated graphene flakes $^{29}$. In order to rule out this mechanism AES was used to determine the thickness of the graphene on the surface before and after cleaning. Figure 4(a) shows the Auger spectrum of a sample without any silver contamination. Clear peaks can be observed at $92 \mathrm{eV}$ and $271 \mathrm{eV}$, corresponding to $\mathrm{Si}$ and graphitic carbon respectively, as expected. A thickness of $3 \pm 1$ graphene layers was estimated based on the C:Si ratio obtained. After contamination and cleaning the Auger spectrum obtained once again exhibited a strong graphitic $\mathrm{C}$ peak at $271 \mathrm{eV}$ and a small Si peak at $92 \mathrm{eV}$ [Fig. 4(b)]. The thickness of the sample after contamination and cleaning, based on the $\mathrm{C}:$ Si ratio, was found once more to be $3 \pm 1$ graphene layers, suggesting that the thickness of the sample did not decrease following contamination being removed from the surface. Repeated deposition and cleaning did not show further changes. STM images showed no evidence of layer removal, with graphene layers still clearly visible after multiple cleaning cycles. The level of damage to the uppermost graphene layer, however, was found to increase slightly after each cycle as expected. Therefore, it was determined that the exfoliation process only removes the contamination from the surface, leaving the uppermost graphene layer intact.

The method discussed has been shown to be effective at removing thick layers of metallic contamination, whilst leaving the graphene layers beneath in good condition. However, in many instances the deposited layer may be thin with only a partial coverage. This type of contamination can often occur as a side effect of different measurement techniques. For example, Fig. 5 shows graphene grown on $\mathrm{SiC}(0001)$, (a) before and (b) after, measurement via AES. It is clear that during this measurement a thin layer of material is now present on the surface. The material shows many similarities to that of samples coated with low coverages of $\mathrm{Ag}$ [Fig. 5(c)], indicating that the new layer is likely to be a thin contamination layer deposited by the electron gun during measurement. The most likely candidate for the source of this material is the $\mathrm{LaB}_{6}$ electron gun filament. The contamination layer was found to cover $46 \%$ of the surface with
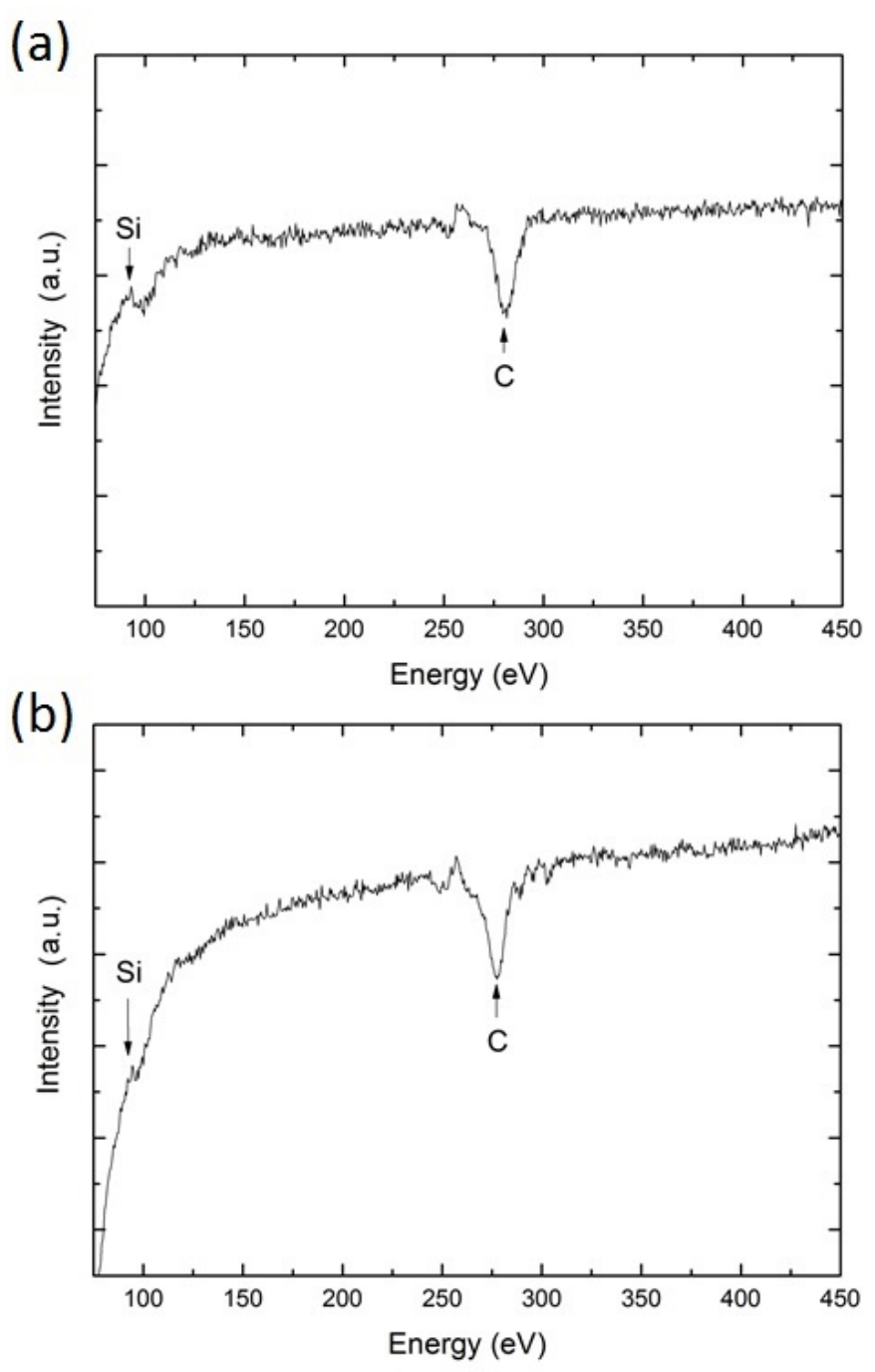

FIG. 4. Auger spectra of graphene on $\mathrm{SiC}(0001)$ taken (a) before contamination with $\mathrm{Ag}$ and (b) after contamination and cleaning. Silicon and carbon peaks are visible in both spectra at $92 \mathrm{eV}$ and $271 \mathrm{eV}$.

a thickness of $\sim 1.2 \mathrm{~nm}$. In earlier studies of graphene on $\mathrm{SiC}(0001)$, similar images have been obtained showing smaller concentrations of this material, which were characterised as roughened graphene caused by hydrogen etching, however, it is possible this is in fact contamination deposited during AES measurements ${ }^{20}$. Adhesive tape was again applied to the sample and removed, to see if this thin contamination layer could also be cleaned. Figure 5(d) shows the graphene surface after mechanical exfoliation of the electron gun contamination. Similarly to the samples contaminated with $\mathrm{Ag},>99 \%$ of the deposited contamination has been removed, leaving the high quality graphene below in excellent condition. In fact, even less damage was observed than previously seen from the removal of the Ag contamination with far fewer instances of small graphene flakes detaching from the surface. Pleat defects on the surface again show changes in 

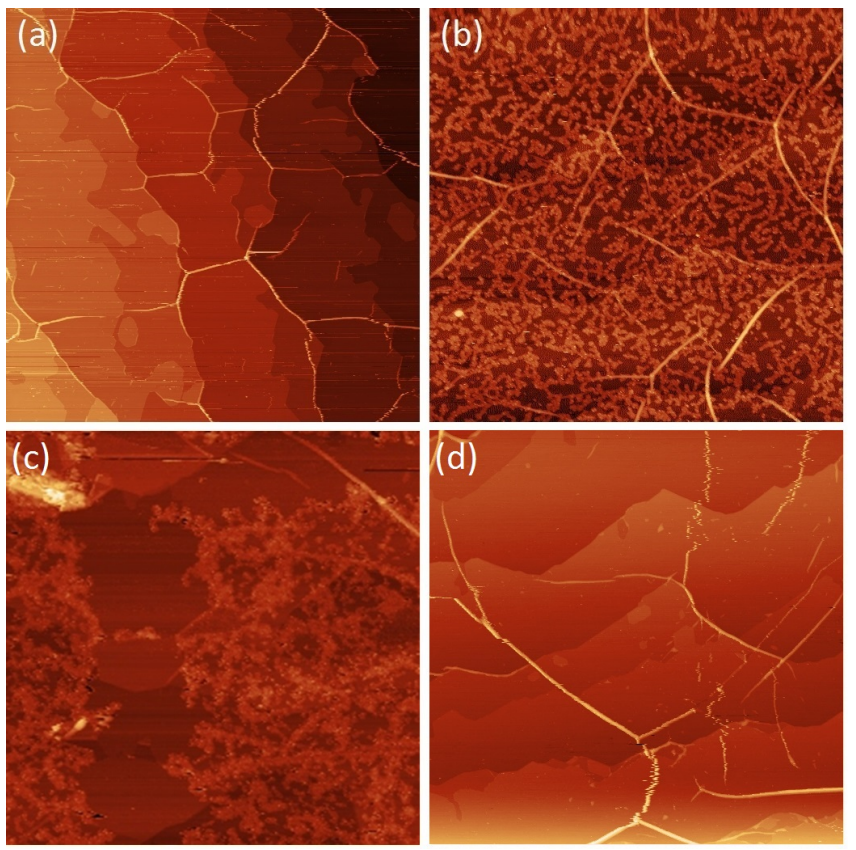

FIG. 5. (Color online) (a) $1 \mu \mathrm{m}^{2}$ STM images of graphene grown on $\mathrm{SiC}$ taken before contamination $\left(\mathrm{V}_{\text {bias }}=1.9 \mathrm{~V}, I=0.3 \mathrm{nA}\right)$ and (b) after contamination from an electron gun $\left(\mathrm{V}_{\text {bias }}=1.0 \mathrm{~V}, I=0.7 \mathrm{nA}\right)$. (c) $500 \mathrm{~nm}^{2}$ image of thin $\mathrm{Ag}$ contamination deposited on graphene grown on $\mathrm{SiC}$, showing similarities to that observed in (b) $\left(V_{\text {bias }}=2.0 \mathrm{~V}, \quad I=0.5 \mathrm{nA}\right) . \quad(\mathrm{d}) \quad 1 \mu \mathrm{m}^{2} \quad \mathrm{STM}$ image of the graphene-SiC sample in (b) after cleaning $\left(\mathrm{V}_{\text {bias }}=0.7 \mathrm{~V}, I=0.4 \mathrm{nA}\right)$.

height, width and length, this as expected from the strain applied to the graphene layers during the cleaning process slightly delaminating areas of the film. Atomic resolution images (not shown) again showed that the lattice remains in pristine condition, indicating that the cleaning process does not cause significant damage to the graphene layers even when removing thin layers of contamination. As seen with deposited $\mathrm{Ag}$, this is likely due to the much stronger adhesion of the graphene layers to the SiC surface compared to the adhesion of the contamination to the uppermost graphene layer ${ }^{22,30}$. The reduced damage observed in this case, compared with samples coated with $\mathrm{Ag}$, is most likely a consequence of the smaller thickness and reduced coverage of the contamination layer. This results in less contamination adhering at reactive sites on the graphene surface, such as defects, where flakes may be pulled up during cleaning. The reactivity of the contamination may play a significant part in the extent of damage caused by this cleaning method. It may be possible that a more reactive metal contaminant, such as $\mathrm{Ni}$, which binds more strongly to the surface ${ }^{31}$, would result in significantly more damage to the uppermost graphene layer. In such a case the method outlined above becomes less suitable as an effective cleaning process.

\section{CONCLUSION}

In conclusion, we have shown that metallic contamination can be removed from graphene layers grown on $\mathrm{SiC}(0001)$ via mechanical exfoliation using low cost commercially available adhesive tape. It was found that by applying adhesive tape and carefully pulling away from the surface $>99 \%$ of contamination could be removed, whilst leaving the graphene layers below in good condition. Only small isolated areas of damage were observed, in which small flakes of graphene were pulled up from the surface. Small changes in the adhesion of the graphene layers to the $\mathrm{SiC}(0001)$ substrate were observed based on the changes to pleat defects observed on the surface, however the atomic scale STM images indicate the graphene remains of excellent quality. Finally, we suggest that material previously characterised as rough graphene ${ }^{20}$ could be associated with contamination deposited during measurement. This contamination could also be removed via the method discussed above, without significant negative effects to the graphene layers below. The method outlined presents a simple, low cost, effective way of removing contamination from graphene grown on $\mathrm{SiC}(0001)$ allowing for their reuse in further experiments.

\section{ACKNOWLEDGEMENTS}

This work was supported by the Leverhulme Trust (Grant No. F/00 125/AN). G.H.W. would like to thank EPSRC for the award of a studentship.

${ }^{1}$ F. Schedin, A. K. Geim, S. V. Morozov, E. W. Hill, P. Blake, M. I. Katnelson, and K. S. Novoselov, Nature Mater. 6, 652 (2007).

${ }^{2}$ Y. Dan, Y. Lu, N. J. Kybert, Z. Luo, and A. T. C. Johnson, Nano Lett. 9, 1472 (2009).

${ }^{3}$ Y. Shao, J. Wang, H. Wu, J. Liu, I. A. Aksay, and Y. Lin, Electroanalysis 22, 1027 (2010).

${ }^{4}$ A. K. Geim and K. S. Novoselov, Nature Mater. 6, 183 (2007).

${ }^{5}$ X. Li, W. Cai, J. An, S. Kim, J. Nah, D. Yang, R. Piner, A. Velamakanni, I. Jung, E. Tutuc, S. K. Banerjee, L. Colombo, and R. S. Ruoff, Science 324, 1312 (2009).

${ }^{6}$ W. A. de Heer, C. Berger, X. Wu, P. N. First, E. H. Conrad, X. Li, T. Li, M. Sprinkle, J. Hass, M. L. Sadowski, M. Potemski, and G. Martinez, Solid State Commun. 143, 92 (2007).

${ }^{7}$ S. Adam, E. H. Hwang, V. M. Galitski, and S. D. Sarma, Proc. Natl. Aca. Sci. U.S.A 104, 18392 (2007).

${ }^{8}$ K. I. Bolotin, K. J. Sikes, Z. Jiang, M. Klima, G. Fudenberg, J. Hone, P. Kim, and H. L. Stormer, Solid State Commun. 146, 351 (2008).

${ }^{9}$ Y. Zhang, V. W. Brar, C. Girit, A. Zettl, and M. F. Crommie, Nature Phys. 5, 722 (2009).

${ }^{10}$ Z. Li, Y. Wang, A. Kozbial, G. Shenoy, F. Zhou, R. McGinley, P. Ireland, B. Morganstein, A. Kunkel, S. P. Surwade, L. Li, and H. Liu, Nature Mater. 12, 925 (2013).

${ }^{11}$ A. Ambrosi, C. K. Chua, B. Khezri, Z. Sofer, R. D. Webster, and M. Pumera, Proc. Natl. Aca. Sci. U.S.A 109, 12899 (2012).

${ }^{12}$ A. Ambrosi and M. Pumera, Nanoscale 6, 472 (2014).

${ }^{13}$ G. Lupina, J. Kitzmann, I. Costina, M. Lukosius, C. Wenger, A. Wolff, S. Vaziri, M. Östling, I. Pasternak, A. Krajewska, 
W. Strupinski, S. Kataria, A. Gahoi, M. C. Lemme, G. Ruhl, G. Zoth, O. Luxemhofer, and W. Mehr, ACS Nano 9, 4776 (2015).

${ }^{14}$ Z. Cheng, Q. Zhou, C. Wang, Q. Li, C. Wang, and T. Fang, Nano Lett. 11, 767 (2011).

${ }^{15}$ Z. H. Ni, H. M. Wang, Z. Q. Luo, Y. Y. Wang, T. Yu, Y. H. Wu, and Z. X. Shen, J. Raman Spectrosc. 41, 479 (2010).

${ }^{16}$ N. Lindvall, A. Kalabukhov, and A. Yurgens, J. Appl. Phys. 111, 064904 (2012).

${ }^{17}$ A. M. Goossens, V. E. Calado, A. Barreiro, K. Watanabe, T. Taniguchi, and L. M. K. Vandersypen, Appl. Phys. Lett. 100, 073110 (2012).

${ }^{18}$ C. A. Joiner, T. Roy, Z. R. Hesabi, B. Chakrabarti, and E. M. Vogel, Appl. Phys. Lett. 104, 223109 (2014).

${ }^{19}$ J. Moser, A. Barreiro, and A. Bachtold, Appl. Phys. Lett. 91, 163513 (2007).

${ }^{20}$ Luxmi, S. N., P. J. Fisher, R. M. Feenstra, G. Gu, and Y.-G. Sun, J. Electron. Mater. 38, 718 (2009).

${ }^{21}$ T. Hopf, K. V. Vassilevski, E. Escobedo-Cousin, P. J. King, N. G. Wright, A. G. O'Neill, A. B. Horsfall, J. Goss, G. H. Wells, and M. R. C. Hunt, J. Appl. Phys. 116, 154504 (2014).

${ }^{22}$ G. H. Wells, T. Hopf, K. V. Vassilevski, E. Escobedo-Cousin, N. G. Wright, A. B. Horsfall, J. P. Goss, A. G. O'Neill, and
M. R. C. Hunt, Appl. Phys. Lett. 105, 193109 (2014).

${ }^{23}$ W. A. de Heer, C. Berger, M. Ruan, M. Sprinkle, X. Li, Y. Hu, B. Zhang, J. Hankinson, and E. Conrad, Proc. Natl. Aca. Sci. U.S.A 108, 16900 (2011).

${ }^{24}$ B. Kiraly, E. V. Iski, A. J. Mannix, B. L. Fisher, M. C. Hersam, and N. P. Guisinger, Nature Commun. 4, 1 (2013).

${ }^{25}$ S. P. Koenig, N. G. Boddeti, M. L. Dunn, and J. S. Bunch, Nature Nanotechnol. 6, 543 (2011).

${ }^{26}$ T. Yoon, W. C. Shin, T. Y. Kim, J. H. Mun, T.-S. Kim, and B. J. Cho, Nano Lett. 12, 1448 (2012).

${ }^{27}$ C. Lee, X. Wei, J. W. Kysar, and J. Hone, Science 321, 385 (2008).

${ }^{28}$ F. Banhart, J. Kotakoski, and A. V. Krasheninnikov, ACS Nano 5, 26 (2011).

${ }^{29}$ K. S. Novoselov, A. K. Geim, S. V. Morozov, D. Jiang, Y. Zhang, S. V. Dubonos, I. V. Grigorieva, and A. A. Firsov, Science 306, 666 (2004).

${ }^{30}$ A. Mattausch and O. Pankratov, Phys.Rev. Lett. 99, 076802 (2007).

${ }^{31}$ J. Lahiri, T. S. Miller, A. J. Ross, L. Adamska, I. I. Oleynik, and M. Batzill, New J. Phys. 13, 025001 (2011). 